\title{
STABILITY OF THE PULSATION OF 12 (DD) LACERTAE
}

\author{
A. PIGULSKI \\ Astronomical Institute, Wroclaw University, Kopernika 11, 51-622 Wroclaw, Poland
}

\begin{abstract}
The results of the study of period changes of all pulsational components in the multiperiodic $\beta$ Cephei-type star, 12 Lac, are summarized.
\end{abstract}

12 (DD) Lacertae is a well-known multiperiodic $\beta$ Cephei-type star. Jerzykiewicz (1978) found 6 short-term periodic components in the variations of light and radial-velocity of the star. Five of them have independent frequencies, the frequency of the last is the sum of two others. Moreover, three of the components form an almost equidistant in frequency triplet, which was usually explained in terms of rotational splitting (Jerzykiewicz 1978, Smith 1980).

Only for the component with largest amplitude $\left(P_{3}=0.19309 \mathrm{~d}\right.$, see Fig.1 for the designation of pulsational components, values of periods, $P$, and frequencies, $f$ ) the changes of the pulsation period were relatively well studied. All these studies indicated that this period decreased, although the last study (Ciurla 1987) found it to be increasing since about 1970.

In the course of our analysis of period changes in $12 \mathrm{Lac}$ we have been able to obtain for the first time the $\mathrm{O}$-C diagrams for all six periodic components (Fig.1). The main results of our study are following:

1. All periods change. The $\mathrm{O}-\mathrm{C}$ diagram for the strongest component $\left(C_{3}\right)$ have a wave-like shape. This confirms the result obtained by Ciurla (1987).

2. $P_{3}$ and $P_{4}$ behave similarly. The changes of $P_{6}$ follow the changes of $P_{3}$ and $P_{4}$, as is expected from the fact that $f_{6}=f_{3}+f_{4}$ is a combinatory frequency.

3. $P_{2}$ changes inversely to $P_{3}$. A similar effect was found by Shobbrook (1972) for two strongest pulsation components of $\beta \mathrm{CMa}$.

4. Neither the evolutionary nor the light-time effect is able to explain the changes of all periods. Even a combination of these two effects does not solve the problem. We conclude therefore, that some unknown effect causes observed period changes. A degree of regularity seen in these changes (points 1-3 above) suggests that this effect probably distinguishes modes with different $l$ and/or $m$.

The full results of this study will be published elsewhere.

\section{Acknowledgements}

This work was supported by the research grant No. 2 P304 00104 from the Polish Scientific Research Committee (KBN), and by a travel grant from the International Astronomical Union. 


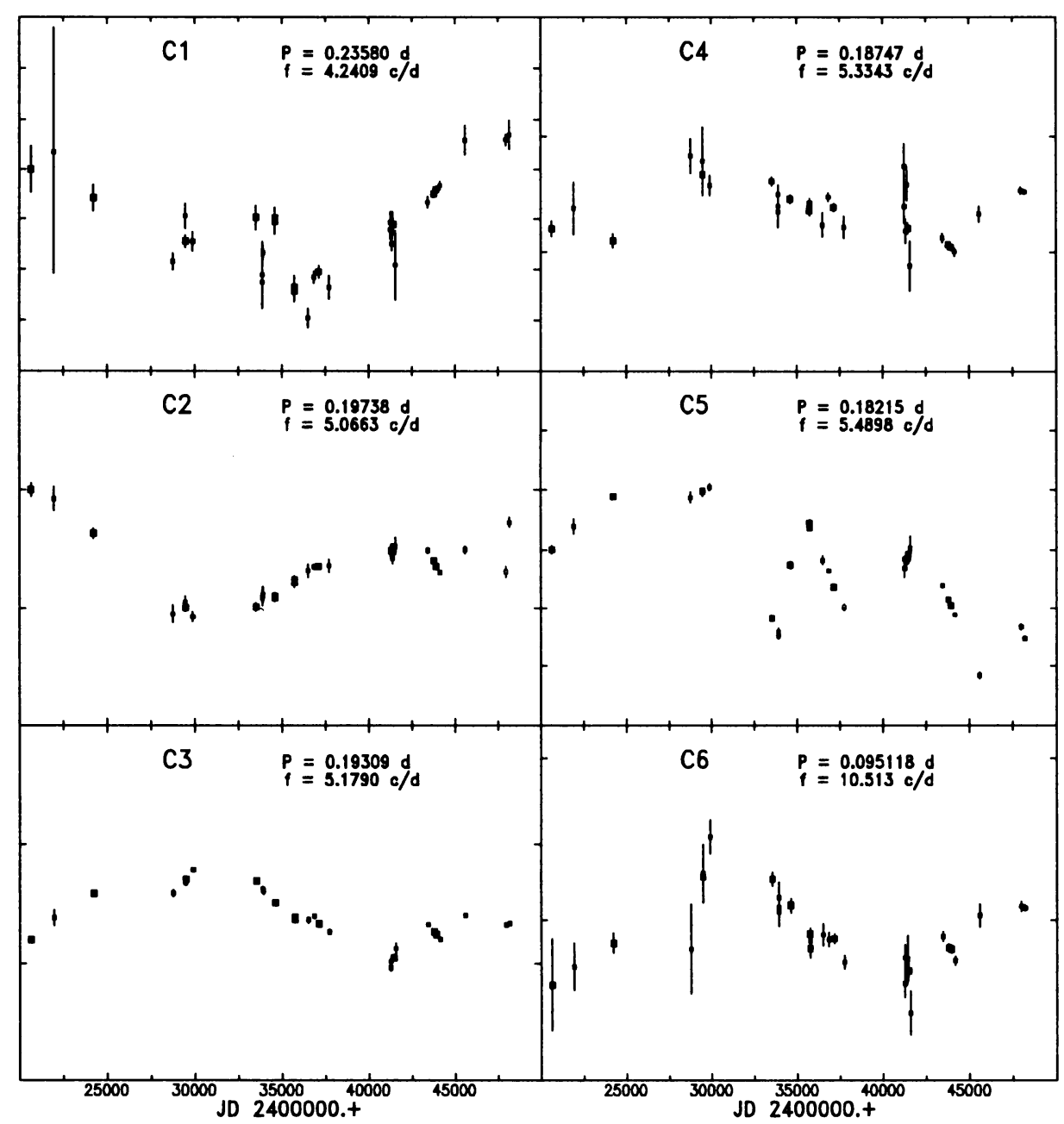

Fig. 1. The O-C diagrams for 6 pulsating components of the light and radial-velocity variations of 12 Lac. For all components the corresponding periods and frequencies are given. The ordinate is $\mathrm{O}-\mathrm{C}$ (in days); the markers are spaced by $0.05 \mathrm{~d}$.

\section{References}

Ciurla, T.: 1987, Acta Astronomica 37, 53

Jerzykiewicz, M.: 1978, Acta Astronomica 28, 465

Shobbrook, R.R.: 1973, M.N.R.A.S. 161, 257

Smith, M.A.: 1980, Ap.J. 240, 149 